\title{
Nonlinear Dynamics Identification of the Oculo-Motor System based on Eye Tracking Data
}

\author{
Vitaliy Pavlenko, Tetiana Shamanina, Vladislav Chori \\ Odessa National Polytechnic University \\ Shevchenko av. 1, Odessa, 65044 \\ Ukraine
}

Received: December 23, 2020. Revised: June 4, 2021. Accepted: June 25, 2021. Published: July 5, 2021.

\begin{abstract}
Instrumental computing and software tools have been developed for constructing a nonlinear dynamic model of the human oculo-motor system (OMS) based on the data of input-output experiments using test visual stimuli and innovative technology eye tracking. For identification the Volterra model is used in the form of multidimensional transient functions of the 1st, 2nd and 3rd orders, taking into account the inertial and nonlinear properties of the OMS. Software tools for processing eye tracking data developed in the Matlab environment are tested on real data from an experimental study of OMS.
\end{abstract}

Keywords - eye tracking technology, identification, oculo-motor system, psycho-physiological states, Volterra model,

\section{INTRODUCTION}

$\mathrm{T}$ HE study of human eye movements and the trajectory of their movement allows us to reveal the structure of the relationship of an individual with the environment, a person with the world. Knowledge about eye movement is of great theoretical and applied importance, expanding the possibilities of studying the specifics of many professions in order to improve the efficiency of the subject of labor activity [1]-[4].

The process of mastering knowledge is a central part of the learning process. Managing this process implies the existence of effective objective indicators for assessing an individual's intellectual abilities. The methods of psychological identification of an individual proposed in the project based on obtaining experimental data using the innovative eye tracking technology and computing means of processing them allow monitoring and diagnostics of the state of cognitive processes during the educational activities of students [5]-[9].
The paper [10] provides an overview of the latest research in the field of physiological systems modeling, as well as advanced methods for analyzing physiological signals and data. The work presented here concerns the development of advanced modeling methodologies and their new application to problems of biomedical interest with an emphasis on nonlinear aspects of physiological function. As the importance of nonlinearities in understanding the complex mechanisms of physiological functions becomes more urgent, the need for effective and practical modeling methodologies that address the problem of nonlinear dynamics in the life sciences becomes more and more relevant.

As a basic model for research, a universal nonlinear dynamic model based on the Volterra integral power series, representing the input-output relationship of the studied physiological system, was chosen.

The aims are to develop instrumental software tools for constructing a nonparametric dynamical model of the OMS human, taking into account its inertial and nonlinear properties, based on data from experimental input-output studies using test visual stimulus and innovative eye tracking technology; implementation of the received information models in practice diagnostics of states cognitive processes.

\section{A. The scope of application}

The developed software enables support of the following tasks:

1) The relationship study of mental states and cognitive processes in educational activities, a post-traumatic stress disorder, the diagnosis of the Parkinson's and Alzheimer's disease stage, checking the psycho-physiological state of pilots and drivers, the professional suitability, the fatigue syndrome [11]-[17].

2) The interaction of mental states and cognitive processes during the educational activities of students, an objective assessment of their cognitive development level, assessment of the effectiveness of training to improve 
mental processes and for psychological correction of personality [17].

3) Extension of the individual's creative life due to the early diagnosis of degenerative processes of cognitive functions of the brain. Identification of a gifted personality (building a psychological model of the personality) and evaluation of its abilities. Professional selection (the identification and education of leaders) [9].

4) The assimilation of scientific knowledge and their respective skills serves as the main goal and the main result of educational activities. The process of mastering knowledge is the central part of the learning process. Managing this process implies the existence of effective objective indicators for assessing an individual's intellectual abilities [7].

The methods of psychological identification of an individual proposed in the project, based on obtaining experimental data using eye tracking technology and computing means of processing them, allow monitoring and diagnostics of the state of cognitive processes during the educational activities of students.

\section{B. Diagnostics neuronal processes}

An intelligent information technology for diagnosing the states of neural processes based on nonparametric identification of OMS in the form of nonlinear dynamic Volterra models is proposed [17] - [19]. The technology involves a consistent solution of the following tasks:

1) Identification of the OMS. The goal is to obtain an OMS information model in the form of MTF. Stages of implementation: the supply of test signals to the inputs of the OMS (horizontally, vertically, diagonally); measurement of OMS responses to test signals with the help of an eye-tracker; MTF calculation based on inputoutput experiment data.

2) Building a diagnostic model of OMS. The goal - the formation of the feature space. Stages of implementation: MTF compression; determination of the diagnostic value of symptoms; selection of the optimal system of signs reduction of the diagnostic model.

3) Building a classifier of the psycho-physiological state of the individual based on the OMS model. The goal is to build a family of decision rules for optimal classification. Stages of implementation: building decision rules based on OMS identification results - training; assessment of the accuracy of the classification - the exam; optimization of the diagnostic model.

4) Diagnostics psycho-physiological state of the personality. The goal is to assess the condition of the individual. Stages of implementation: OMS identification; evaluation of diagnostic signs; classification - the assignment of the investigated individual to a particular class.

\section{THE POLYNOMIAL VOLTERRA MODEL}

The input-output ratio for a nonlinear dynamical system (NDS) with an unknown structure (such as a "black box") with a single input and a single output can be represented by a discrete cubic Volterra polynomial in the form [20], [21]:

$$
\begin{aligned}
& y[m]=\sum_{n=1}^{3} y_{n}[m]=\sum_{k_{1}=0}^{m} w_{1}\left[k_{1}\right] x\left[m-k_{1}\right]+ \\
& +\sum_{k_{1}=0}^{m} \sum_{k_{2}=0}^{m} w_{2}\left[k_{1}, k_{2}\right] x\left[m-k_{1}\right] x\left[m-k_{2}\right]+ \\
& +\sum_{k_{1}=0}^{m} \sum_{k_{2}=0}^{m} \sum_{k_{3}=0}^{m} w_{3}\left[k_{1}, k_{2}, k_{3}\right] x\left[m-k_{1}\right] x\left[m-k_{2}\right] x\left[m-k_{3}\right],
\end{aligned}
$$

where $w_{1}\left[k_{1}\right], w_{2}\left[k_{1}, k_{2}\right], w_{3}\left[k_{1}, k_{2}, k_{3}\right]$ - discrete weight functions (Volterra kernels) of the 1st, 2nd and 3rd orders; $x[m], y[m]-$ input (stimulus) and output (response) function (signals) of the system, respectively; $y_{n}[\mathrm{~m}]$ - partial components of the response (convolution of $n$-th order sequences); $m$ is a discrete time variable.

The problem of identification is to choice test signals $x[\mathrm{~m}]$ and develop an algorithm that allows based on the responses received $y[m]$ to identify partial components $y_{n}[m],(n=1,2$, 3 ) and determine on their basis multidimensional Volterra kernels: $w_{1}\left[k_{1}\right], w_{2}\left[k_{1}, k_{2}\right], w_{3}\left[k_{1}, k_{2}, k_{3}\right][19]$.

Taking into account the specifics of the studied OMS, test step signals are used for identification. If the test signal $x[m]=\theta[m]$, where $\theta[m]$ is a unit function (Heaviside function), then the partial components of the response $y_{1}[\mathrm{~m}], y_{2}[\mathrm{~m}], y_{3}[\mathrm{~m}]$ will be equal to the transient function of the first order $h_{1}[\mathrm{~m}]$ and diagonal sections of the transient functions of the second and third orders $h_{2}[m, m], \quad h_{3}[m, m, m]$ respectively [22], [23]:

$$
\begin{aligned}
& y_{1}[m]=h_{1}[m]=\sum_{k_{1}=0}^{m} w_{1}\left[m-k_{1}\right], \\
& y_{2}[m]=h_{2}[m, m]=\sum_{k_{1}=0}^{m} \sum_{k_{2}=0}^{m} w_{2}\left[m-k_{1}, m-k_{2}\right], \\
& y_{3}[m]=h_{2}[m, m, m]=\sum_{k_{1}=0}^{m} \sum_{k_{2}=0}^{m} \sum_{k_{3}=0}^{m} w_{3}\left[m-k_{1}, m-k_{2}, m-k_{3}\right] .
\end{aligned}
$$

Determination of subdiagonal intersections of transient functions is based on the NDS test using $L$ test step signals with given amplitudes $a_{i}, i=1,2, \ldots, L(L>=N, N$ is the degree of the Volterra polynomial). In this case the responses of the NDS are denoted by $y_{1}[m], y_{2}[m], \ldots, y_{L}[m]$. Reviews of the Volterra model will be view

$$
\tilde{y}_{i}[m]=a_{i} \hat{y}_{1}[m]+a_{i}^{2} \hat{y}_{2}[m]+a_{i}^{3} \hat{y}_{3}[m], i=\overline{1, L},
$$

where $\quad \hat{y}_{1}[m]=\hat{h}_{1}[m], \hat{y}_{2}[m]=\hat{h}_{2}[m, m], \hat{y}_{3}[m]=\hat{h}_{3}[m, m, m]$ obtained estimates of the partial components of the model multidimensional transient functions.

To determine the transient functions $h_{1}[m], h_{2}[m, m], h_{3}[m, m, m]$ is used the method of least squares (LSM), which provides the minimum standard error of the deviation of the model responses from the responses of the OMS to the same stimulus:

$$
J_{N}=\sum_{j=1}^{L}\left(y_{j}[m]-\sum_{n=1}^{N} a_{j}^{n} \hat{y}_{n}[m]\right)^{2} \rightarrow \min .
$$

The minimization of criterion (4) is reduced to solving a system of normal Gaussian equations, which in vector-matrix form can be written as

$$
\mathrm{A}^{\prime} \mathrm{A} \hat{y}=\mathrm{A}^{\prime} \mathrm{y}
$$


where $\mathrm{A}=\left\|\alpha_{i j}\right\|, \alpha_{i j}=a_{i}^{j}, i, j=\overline{1, N}$.

The system of Gaussian normal equations (5) produces good results on the approximation of functions if the number of measurements $L$ is large enough (much greater than the degree of the approximating polynomial $N$ ) or the measurement errors are small. Otherwise, the determinant of the system turns out to be close to zero and the system becomes ill-conditioned. In this case, large errors in the parameters estimation of the approximating polynomial are possible.

Tikhonov's method of regularization [24], which is based on a variational method for constructing a regularizing operator, is used to obtain a solution of linear algebraic equations system (5) that is stable against measurement errors. This method is reduced to finding an approximate solution vector $\hat{\mathrm{y}}_{\alpha}$ that minimizes certain smoothing functional. The only vector satisfying the condition of the smoothing functional minimum can be determined from the solution of linear algebraic equations system:

$$
\left(\mathrm{A}^{\prime} \mathrm{A}+\alpha \mathrm{I}\right) \hat{\mathrm{y}}_{\alpha}=\mathrm{A}^{\prime} \mathrm{y},
$$

where $\mathrm{A}^{\prime}$ is the transposed matrix; I is the identity matrix; $\alpha$ is the Tikhonov regularization parameter.

The approximate solution obtained on the basis of (6) corresponds to the zero order of regularization (weak regularization). To increase of the smoothness solutions, the regularization matrix $\mathrm{R}$ is used and the solution of the system of linear algebraic is calculated

$$
\left(\mathrm{A}^{\prime} \mathrm{A}+\alpha \mathrm{R}\right) \hat{\mathrm{y}}_{\alpha}=\mathrm{A}^{\prime} \mathrm{y}
$$

with the selected value of the parameter $\alpha$. The regularization matrix $\mathrm{R}$ has a tape structure (8):

$$
R=\left[\begin{array}{ccccccc}
1-h^{-2} & -\frac{1}{h^{2}} & 0 & \cdots & 0 & 0 & 0 \\
-\frac{1}{h^{2}} & 1-h^{-2} & -\frac{1}{h^{2}} & 0 & 0 & 0 & 0 \\
0 & -\frac{1}{h^{2}} & 1-h^{-2} & -\frac{1}{h^{2}} & 0 & 0 & 0 \\
\vdots & 0 & -\frac{1}{h^{2}} & \ddots & -\frac{1}{h^{2}} & 0 & \vdots \\
0 & 0 & 0 & -\frac{1}{h^{2}} & 1-h^{-2} & -\frac{1}{h^{2}} & 0 \\
0 & 0 & 0 & 0 & -\frac{1}{h^{2}} & 1-h^{-2} & -\frac{1}{h^{2}} \\
0 & 0 & 0 & \ldots & 0 & -\frac{1}{h^{2}} & 1-h^{-2}
\end{array}\right]
$$

where $h$ is the second regularization parameter, usually coincides with the amplitude sampling step of the test signals $\Delta a$.

When implementing this algorithm, the regularization parameter $\alpha$ is chosen sufficiently small from the analysis of the available information about the error of the initial data and the calculation error. In the work, the appropriate value of the regularization parameter $\alpha$ is determined by selection, i.e. repeated calculations $\hat{\mathrm{y}}_{\alpha}$, for different values of $\alpha$. The quasioptimal value of the parameter $\alpha=\alpha_{0}$ is selected from the condition

$$
\left\|\hat{\mathrm{y}}_{\alpha \mathrm{i}+1}-\hat{\mathrm{y}}_{\alpha \mathrm{i}}\right\| \rightarrow \min ,
$$

where $\alpha_{i+1}=\mu \alpha_{i}, 0<\mu<1, i=0,1,2, \ldots$ It should be noted that different ways of determining the regularization parameter can give different results and, as a consequence, different regularized solutions.

Another way to ensure the computational stability of linear algebraic equations system solutions is the preprocessing of eye tracking data using Kalman filtering [25] and Lainiotis Information Filter [26].

\section{COMPUTING OF TRANSIENT FUNCTIONS OMS}

Information technology of the constructing a nonparametric dynamic model of the human OMS taking into account its inertial and nonlinear properties based on the data of experimental studies input-output was developed. As a basic OMS model - the Volterra model is used in the form of multidimensional transient functions.

Methods and tools for the identification of OMS have been developed using the help of eye tracking technology, and building a features space and optimal classification human states using machine learning. In the Laboratory of Motion Analysis and Interface Ergonomics at the Lublin University of Technology (Lublin, Poland), joint studies of the human OMS were performed to obtain diagnostic information for solving urgent problems in the neuro informatics and the computational neuroscience. Experimental research was carried out using eye tracking technology with the use of the video based Tobii TX300 (300 Hz sampling rate) eye tracker and appropriate software [17].

The following instrumental algorithmic and software tools are developed to achieve the goal of the research:

1) Formation of test signals in the form of bright dots on the computer monitor screen at different distances from the initial position horizontally, vertically and diagonally.

2) Preprocessing (bringing the OMS responses to a common start and rationing to one) and analyzing the data obtained from the eye tracker.

3) Constructing an identification model of OMS in the form of multidimensional transient functions (integral transformations of Volterra kernels).

4) Visualization of data and processing results of experimental research.

\section{A. Experimental research of the OMS}

When conducting experimental studies, such actions are carried out:

1) The test subject is placed in front of the computer so that his eyes are at the center of the monitor at a distance of 40-50 cm from him.

2) The subject's head is fixed in order to prevent its movements during the study and to ensure the same experimental conditions.

3) On the subject's readiness, the Signal Manager of the test visual stimulus program is launched.

4) A red circle appears in the center (or from its edge) - of the screen in the starting position.

5) After a short pause (2-3 sec.), the circle in the starting position disappears and a circle of a different color appears at the point with the specified coordinates - a visual stimulus (test signal), which is displayed in this 
position for a specified duration (1-2 sec.) - the action makes the eye move in the direction of the visual stimulus.

6) Then this stimulus circle disappears and a red circle appears in the starting position - this makes the eye move in the opposite direction to the starting position, after these actions the experiment ends.

7) Using the eye tracker, the coordinates of the pupil of the eye are determined during its movement (reaction to the visual stimulus) in the period between the starting positions and the coordinate values are stored in the xlsfile.

In the studies of each respondent, three experiments were successively implemented for three amplitudes of test signals in the horizontal direction. The distance between the starting position and the test incentives is equal to: $0.33 l_{x}, 0.66 l_{x}, 1.0$ $l_{x}$, where $l_{x}$ is the length of the monitor screen. Coordinates of the starting position $\left(x=0, y=0.5 l_{y}\right), l_{y}$ - mean the width of the monitor screen.

The obtained results of measurements of the OMS responses at $L=3$ obtained with using the Tobii TX300 eye tracker in one study cycle ("Horizontally") are shown in Fig.1. Transient process in the OMS response to the test signal $a_{1}=0.33 l_{x}$ are illustrated on Fig. 2.

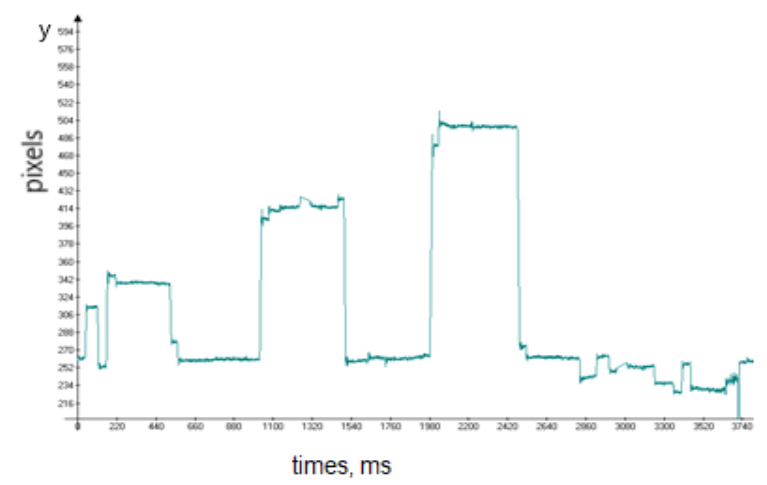

Fig. 1 the OMS responses at $L=3$ obtained using the Tobii TX300 eye-tracker

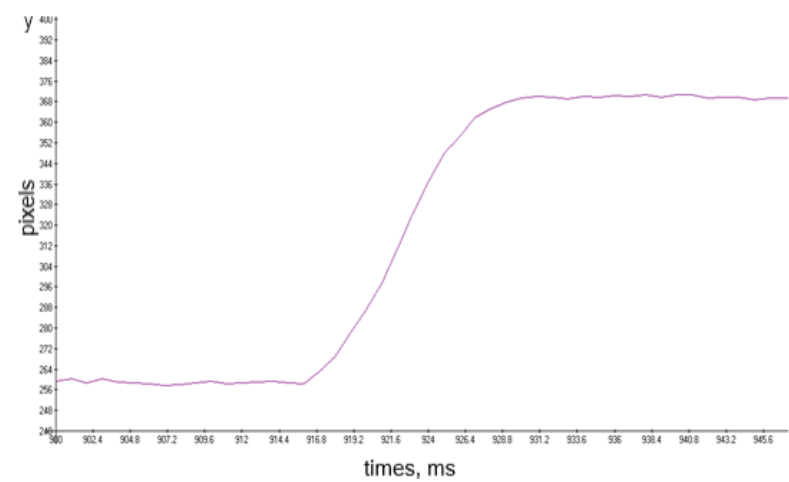

Fig. 2 transient process in the OMS response to the test signal: $a_{1}$

\section{RESEARCH RESULTS}

The experiments were organized in order to classify subjects by the state of fatigue. The data for constructing the model the OMS responses to the same test signals, were obtained using the Tobii Pro TX300 eye tracker at different times of the day: "In the Morning" (before work) and "In the Evening" (after work). The average values of the OMS responses obtained from the eye tracker at various amplitudes of the test signals "In the Morning" and "In the Evening" are shown in Fig. 3.

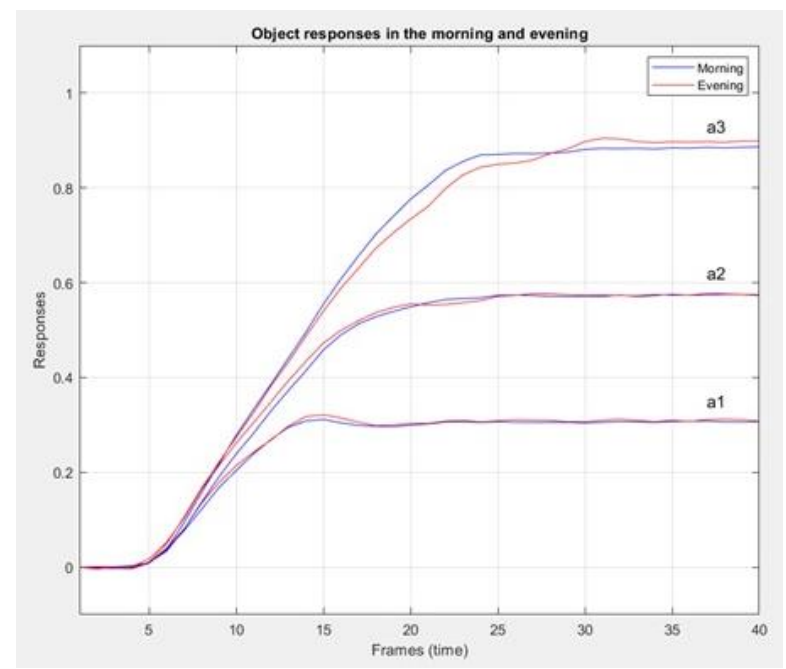

Fig. 3 averaged OMS responses at various amplitudes of test signals "In the Morning" and "In the Evening"

According to averaged data of OMS responses on visual stimuli with a different distance from the start position on the basis of formula (5) the functions of the OMS were defined when approximation models of degrees $N=3$ were used. Graphs of the transient functions estimates for the "In the Morning" and "In the Evening" states of the subject based on model (1) are shown in Fig. 4.

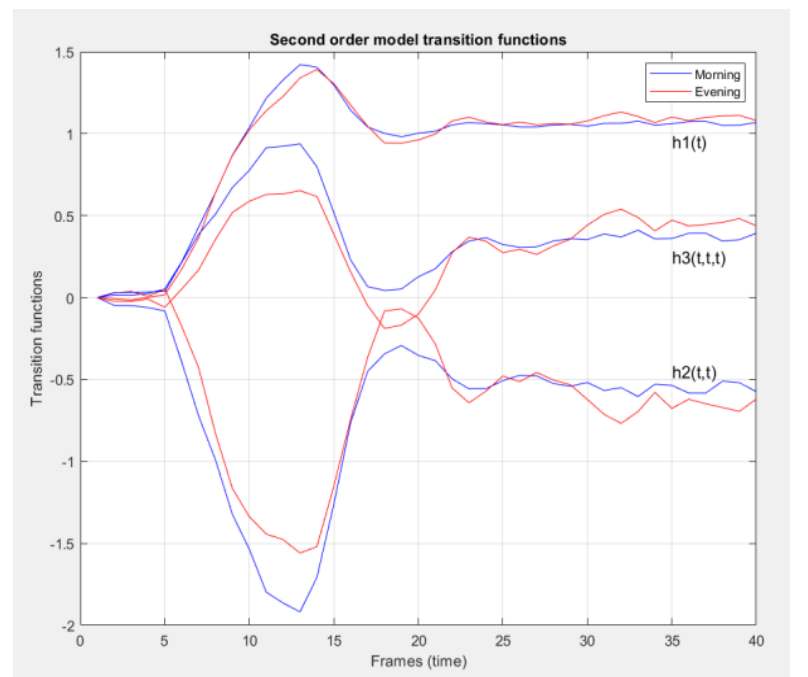

Fig. 4 the transient functions estimates of the 1st, 2nd, and 3rd orders $(N=3)$ for states of the test subject "In the Morning" and "In the Evening"

Received responses with the help of calculations on models at $N=3$ from various amplitudes of test signals "In the Morning". Graphs these are presented in comparison with similar responses OMS in Fig. 5. Graphs of responses of the model OMS at $N=3$ at various amplitudes of the test signals "In the Morning" and "In the Evening" are illustrated in Fig. 6. 


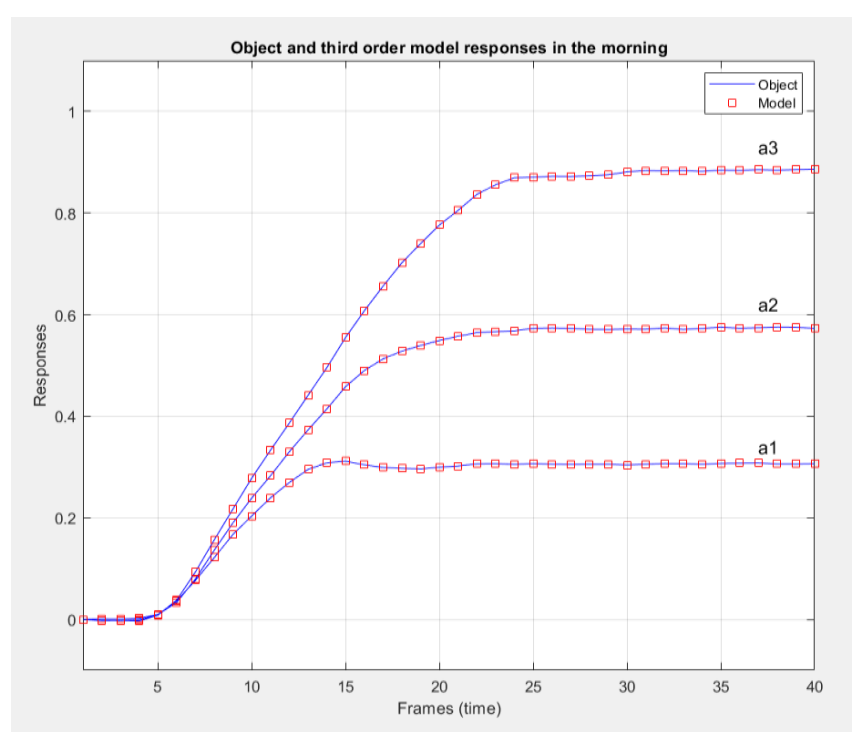

Fig, 5 the responses of the OMS and the model at $N=3$ at various amplitudes of the test signals "In the Morning"

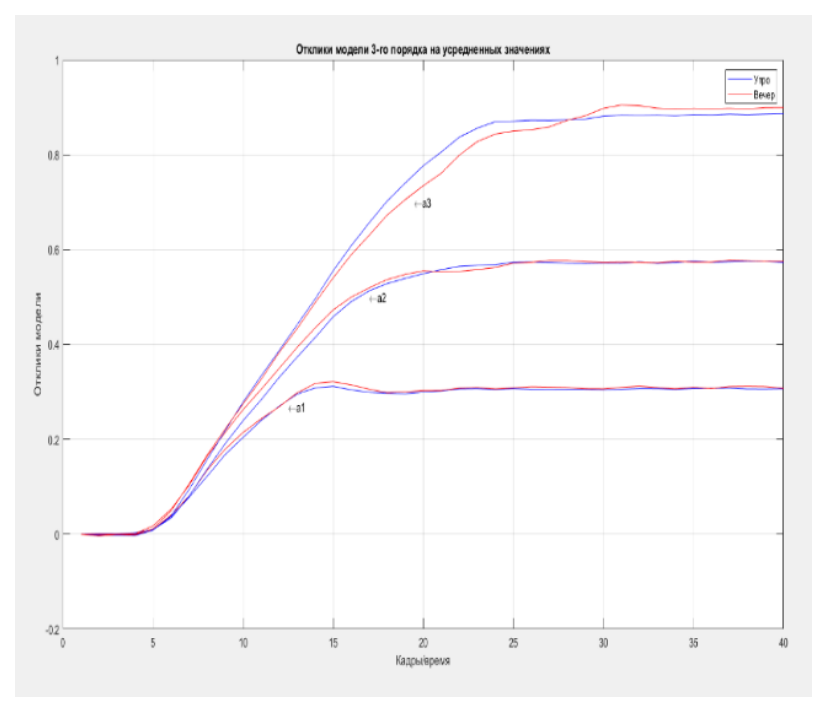

Fig. 6 the responses of the model at $N=3$ at various amplitudes of the test signals "In the Morning" and "In the Evening"

\section{A. Deviation of the transient functions}

The variability (deviation) of the MTF of different orders $n$ of the approximation model of OMS for the states of the respondent "In the Morning" and "In the Evening" is quantified using the indicator of $\varepsilon_{\mathrm{nN}}$ - normalized standard deviation (10). The indicators deviation of the MTF of different orders $n$ of the OMS approximation model for respondent states "In the Morning" and "In the Evening" are given in Table 1 and are represented by diagram in Fig. 7.

$$
\varepsilon_{n N}=\left(\frac{\sum_{m=0}^{M}\left(\hat{y}_{n e}[m]-\hat{y}_{n m}[m]\right)^{2}}{\sum_{m=0}^{M}\left(\hat{y}_{n m}[m]\right)^{2}}, n=\overline{1, N}\right.
$$

Table 1. The deviation indicators of multidimensional transient functions

\begin{tabular}{|c|c|c|c|}
\hline$N$ & $\varepsilon_{1 \mathrm{~N}}$ & $\varepsilon_{2 \mathrm{~N}}$ & $\varepsilon_{3 \mathrm{~N}}$ \\
\hline 1 & 0.019 & - & - \\
\hline 2 & 0.051 & 0.232 & - \\
\hline 3 & 0.04 & 0.199 & 0.322 \\
\hline
\end{tabular}

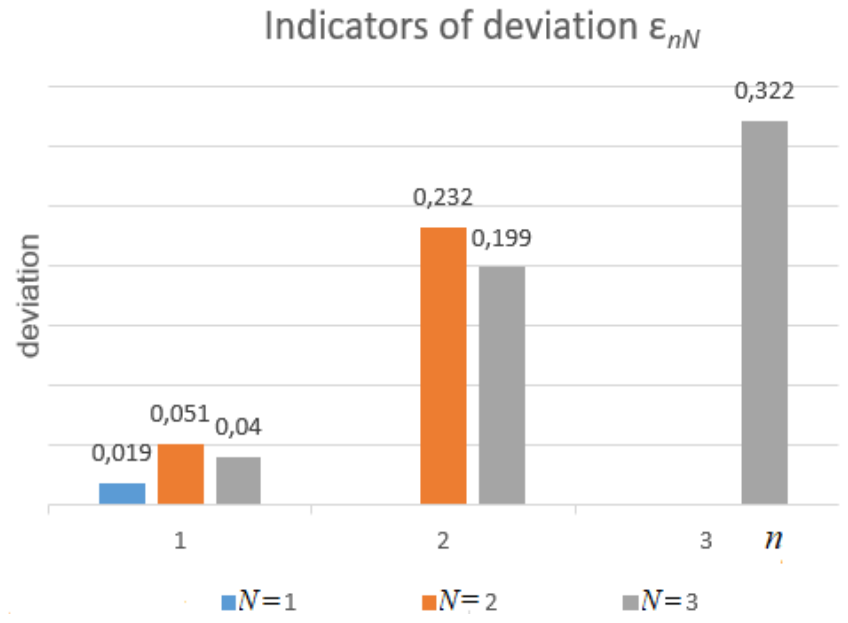

Fig. 7 the diagram of deviations indicators $\varepsilon_{n N}$

As can be seen from figure 5 , the obtained transient function of the 1st order for the "In the Morning" and "In the Evening" are virtually independent of the status of the subject. However, the diagonal cross section of the transient functions of the second and third order change significantly in magnitude and, therefore, can be effectively used as the primary data source when building models of classifiers of psychophysiological conditions of the person using machine learning.

\section{B. Building a classifier of the fatigue}

To assess the psycho-physiological state of a person based on the OMS model, studies were carried out:

1) Getting a feature space for building a human state classifier using machine learning [27] - [29].

2) Building classifiers using deterministic and statistical learning methods for pattern recognition based on the data obtained using eye tracking technology.

The discriminant function $d(\mathrm{x})$ is sequentially calculated on the basis of training datasets for object classes A ("In the Morning"), B ("In the Evening"). To separate the two classes (dichotomy case) a discriminant function of the form is used:

$$
\begin{aligned}
& d(\mathrm{x})=\frac{1}{2} \mathrm{x}^{\prime}\left(\mathrm{S}_{2}^{-1}-\mathrm{S}_{1}^{-1}\right) \mathrm{x}+\left(\mathrm{S}_{1}^{-1} \mathrm{~m}_{1}-\mathrm{S}_{2}^{-1} \mathrm{~m}_{2}\right)^{\prime} \mathrm{x}+ \\
& +\frac{1}{2}\left(\mathrm{~m}_{1}^{\prime} \mathrm{S}_{1}^{-1} \mathrm{~m}_{1}-\mathrm{m}_{2}^{\prime} \mathrm{S}_{2}^{-1} \mathrm{~m}_{2}+\ln \frac{\left|\mathrm{S}_{2}\right|}{\left|\mathrm{S}_{1}\right|}\right)+\lambda_{\max }
\end{aligned}
$$

where $\mathrm{x}=\left(x_{1}, x_{2}, \ldots, x_{n}\right)^{\prime}-$ features vector, $n$ - features space dimensionality, $\mathrm{m}_{i}$ - mathematical expectation vector for a features of class $i, i=1,2 ; \mathrm{S}_{i}=\mathrm{M}\left[\left(\mathrm{x}-\mathrm{m}_{i}\right)\left(\mathrm{x}-\mathrm{m}_{i}\right)^{\prime}\right]$ - covariance matrix for class $i(\mathrm{M}[]-$ mathematical expectation operation). $\mathrm{S}_{i}^{-1}$ - matrix inverse to $\mathrm{S}_{i},\left|\mathrm{~S}_{i}\right|$ - matrix determinant $\mathrm{S}_{\mathrm{i}}, \lambda_{\max }-$ classification threshold providing the highest criterion probability of correct recognition training sample objects. 
The analysis of the quality of various features combination is carried out on the basis of the criterion probability of correct recognition (PCR, $P$ ). The quality of the combination of the selected features from the considered set of features is assessed based on the classification results on the studied data sample.

Bayesian classifier. Bayesian classifier of a person's fatigue state in a two-dimensional feature space is provided with the maximum recognition reliability $(P=0.9375)$ with combinations of the following features:

$$
\begin{aligned}
& \left(x_{3}=\sum_{m=1}^{M}\left|h_{3}(m, m, m)\right| \& x_{14}=\underset{m}{\min } \underset{2}{h_{2}^{\prime}}(m, m)\right) \text { or } \\
& \left(x_{3}=\sum_{m=1}^{M}\left|h_{3}(m, m, m)\right| \& x_{15}=\underset{m}{\min } h_{3}^{\prime}(m, m, m)\right) \text { or } \\
& \left(x_{6}=\arg \max \underset{m}{h_{3}^{\prime}}(m, m, m) \& x_{11}=\underset{m}{\max } \underset{h_{2}^{\prime}}{\prime}(m, m)\right) \text { or } \\
& \left(x_{8}=\arg \min h_{m}^{\prime}(m, m) \& x_{11}=\underset{m}{\max } h_{2}^{\prime}(m, m)\right) \text { or } \\
& \left(x_{14}=\underset{m}{\max } h_{2}^{\prime}(m, m) \& x_{15}=\underset{m}{\max } h_{3}^{\prime}(m, m, m)\right) \text { or } \\
& \left(x_{14}=\underset{m}{\min } h_{2}^{\prime}(m, m) \& x_{15}=\underset{m}{\min } h_{3}^{\prime}(m, m, m)\right) \text {. }
\end{aligned}
$$

Separately, the PCR features: $x_{6}$ or $x_{8}-P=0,625 ; x_{3}$ or $x_{12}-$ $\mathrm{P}=0,6875 ; x_{14}-\mathrm{P}=0,75 ; x_{11}$ or $x_{15}-P=0,8125$.

Support Vector Machine classifier. Support Vector Machine (SVM) [30] classification gives close results $(P=0,875)$ in two-dimensional feature space $x_{6}, x_{11}$ or $x_{6}, x_{12}$. The classifier is built using a 2 nd order polynomial kernel:

$$
K\left(\mathrm{x}, \mathrm{x}^{\prime}\right)=\left(\left\langle\mathrm{x}, \mathrm{x}^{\prime}\right\rangle+1\right)^{d}
$$

where $d$ is specified by parameter degree, $d=2$.

Scikit-learn library was used (class sklearn.svm.SVC) to apply SVM.

The location of the objects of the training sample in the space of features $x_{6}, x_{11}$ and $x_{6}, x_{12}$ is shown in Fig. 8 and 9 accordingly.

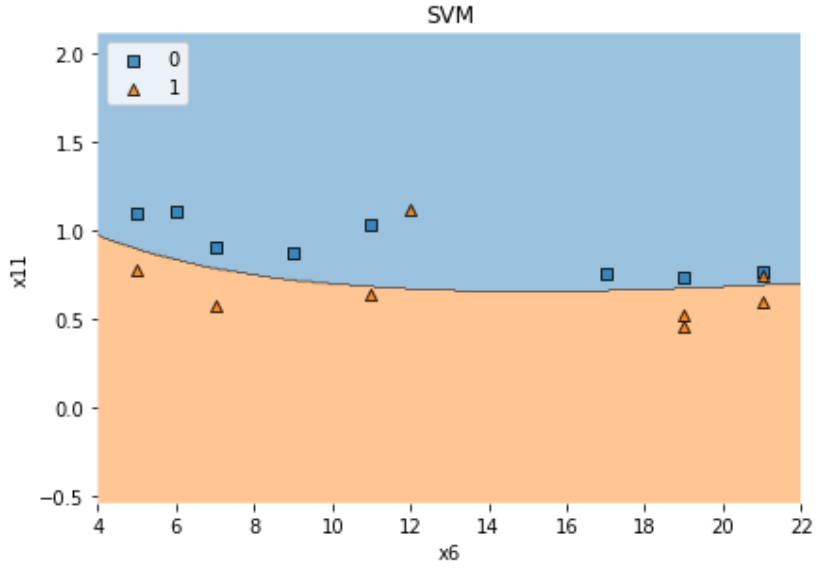

Fig. 8 the location of the objects of the training set in the space of features $x_{6}$ and $x_{11}$

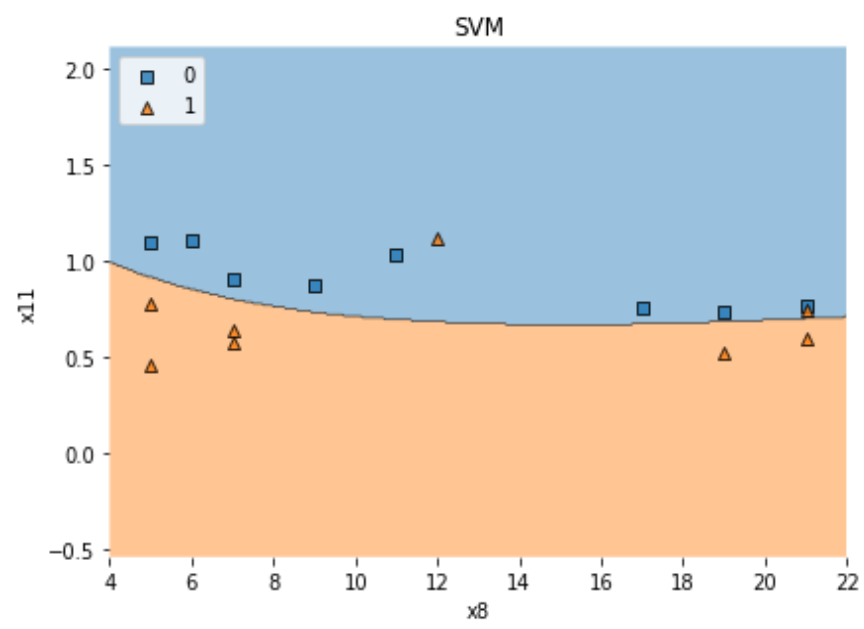

Fig. 9 the location of the objects of the training set in the space of features $x_{8}$ and $x_{11}$

\section{CONCLUSION}

A method and software for constructing a nonparametric dynamic human OMS model have been developed, taking into account its inertial and nonlinear properties, based on experimental input-output data using test visual stimuli and innovative eye tracking technology. A nonlinear dynamic model in the form of a Volterra polynmial is used.

Test visual stimulus are used in the form of bright points, which are sequentially displayed at different distances from the initial position. This formally corresponds to the different amplitudes of the step test signals. The transient functions of the 1st, 2nd, and 3rd orders are determined using LSM.

The developed software tools for eye tracking data processing are tested on real data from the OMS experimental studies. Verification of the constructed model confirms the adequacy of the studied OMS model - a practical coincidence (within an acceptable error) of the OMS responses and its model to the same test signal.

The variability of the transient functions of the 2nd and 3rd orders for different psychophysiological states of the respondent (the level of fatigue) was revealed. Thus, they can be used in diagnostic studies in the field of neuroscience and psychology.

\section{References}

[1] Y. Wamain, X. Corveleyn, L. Ott, Y. Coello, "Does the motor system contribute to the perception of changes in objects visual attributes? The neural dynamics of sensory binding by action," Neuropsychologia, 132, 2019. 107121.doi.org/10.1016/j.neuropsychologia.2019.107121

[2] M. Daoudi, M. Coello, P. Desrosiers, L. Ott, "A New Computational Approach to Identify Human Social Intention in Action," In the 13th IEEE International Conference on Automatic Face \& Gesture Recognition, FG 2018, Xi'an, China on May 15-19. (2018).

[3] F. Quesque, A. Mignon, Y. Coello, "Cooperative and competitive contexts do not modify the effect of social 
intention on motor action. Consciousness and Cognition. doi: 10.1016/j.concog," 2017, 06.011.

[4] L. Lanata, Sebastian, F. Di Gruttola, S. Di Modica, E.P. Scilingo and A. Greco1 "Nonlinear Analysis of EyeTracking Information for Motor Imagery Assessments. Frontiers in Neuroscience", 2020, 13:1431. DOI: 10.3389/fnins.2019.01431.

[5] N. Gueugneau, L.Crognier, and C. Papaxanthis, "The influence of eye movements on the temporal features of executed and imagined arm movements," Brain Research, 1187, 2008, pp. 95-102.

[6] A. Guillot, C. Collet, "Duration of mentally simulated movement: A review,' Journal of Motor Behaviour, 2005, 37, pp.10-20.

[7] A. Guillot, C. Collet, "Construction of the motor imagery integrative model in sport: A review and theoretical investigation of motor imagery use," International Review of Sport and Exercise Psychology, 1, 2008, pp. 32-44.

[8] A. Guillot, C. Collet, V.A. Nguyen, F. Malouin, C. Richards, and J. Doyon, "Functional neuroanatomical networks associated with expertise in motor imagery ability," Neuroimage, 41, 2008, pp. 1471-1483.

[9] A. Guillot, M. Louis, and C. Collet, "Neurophysiological substrates of motor imagery ability," In A. Guillot and C. Collet (Eds). The neurophysiological foundations of mental and motor imagery. Oxford University Press, 2010, pp. 109-124.

[10] R.A. Sandler, S.A. Deadwyler, R.E. Hampson, DongSong, T.W. Berger, V.Z. Marmarelis, "System identification of point-process neural systems using Probability Based Volterra kernels," J Neurosci Methods, 2015, 240, pp 179-192.

[11]M. El Haj, Y. Coello, D. Kapogiannis, K. Gallouj, P. Antoine, "Negative prospective memory in Alzheimer's Disease: do not perform that action," Journal of Alzheimer's Disease, 2018, 61(2), pp. 663-672, doi: 10.3233/JAD-170807.

[12] X. Corveleyn, J. Blampain, L. Ott, I. Lavenu, C. Delayen, A. Di Pastena, Y. Coello, "Body-centred and objectcentred motor imagery in Alzheimer disease", 2018, Current Alzheimer Research, 15 (3), doe: 10.2174/156720 504666171030105720 .

[13] D Jansson, A. Medvedev, H. Axelson and D. Nyholm "Stochastic anomaly detection in eye tracking data for quantification of motor symptoms in Parkinson's disease", Advances in Experimental Medicine and Biology, 2015, 823 pp. 63-82.

[14] D. Jansson, O. Rosén and A. Medvedev, "Parametric and nonparametric analysis of eye-tracking data by anomaly detection," IEEE Transaction control system technology, 23, 2015, pp. 1578-1586.

[15] V. Bro and A. Medvedev "Nonlinear dynamics of the human smooth pursuit system in health and disease: model structure and parameter estimation", IEEE 56th Annual Conference on Decision and Control, (Melbourne) 46924697, 2017.

[16] I. Rigas, O. Komogortsev and R. Shadmehr, "Biometric recognition via the complex eye movement behavior and the incorporation of saccadic vigor and acceleration cues," ACM Trans. on Applied Perception, 2016, 13 (2), pp.121.

[17] V.D. Pavlenko, M. Milosz and M. Dzienkowski, "Identification of the oculo-motor system based on the Volterra model using eye tracking technology," 4th Int. Conf. on Applied Physics, Simulation and Computing (APSAC 2020) 23-25 May 2020, Rome, Italy, Journal of Physics: Conference Series, Volume 1603, 2020 , IOP Publishing, 2020, pp. 1-8, doi:10.1088/17426596/1603/1/012011.

[18] V. Pavlenko, D. Salata, M. Dombrovskyi and Y. Maksymenko, "Estimation of the multidimensional transient functions oculo-motor system of human," AIP Conf. Proc. 1872, 2017, pp.110-117.

[19] V. Pavlenko, D. Salata, and H. Chaikovskyi, "Identification of a oculo-motor system human based on Volterra kernels," International Journal of Biology and Biomedical Engineering, 11, 2017, pp.121-126.

[20]F.J. Doyle, R.K. Pearson and B.A. Ogunnaike, "Identification and control using Volterra models", Springer Publ, 2002, p. 314.

[21] V. Pavlenko, S. Pavlenko, V. Speranskyy, "Identification of Systems using Volterra Model in Time and Frequency Domain," In book: «Advanced Data Acquisition and Intelligent Data Processing». Chapter 10. V. Haasz and K. Madani (Eds.) - River Publishers, 2014, pp.233-270, ISBN 978-87-93102-73-6.

[22] V. Pavlenko, I. Ivanov, and E. Kravchenko, "Estimation of the multidimensional dynamical characteristic eyemotor system," Proceedings of the 9th IEEE International Conference on Intelligent Data Acquisition and Advanced Computing Systems: Technology and Applications, (Bucharest), 2, 2017, pp. 645-650.

[23] A. Fomin, M. Masri, V. Pavlenko, and A. Fedorova, "Method and Information Technology for Constructing a Nonparametric Dynamic Model of the Oculomotor System," Eastern European Journal of Enterprise Technologies. ,2015, №2/9(74), pp. 64-69, DOI: 10.15587/1729-4061.2015.41448.

[24] A. Tikhonov, A. Goncharsky, V. Stepanov, and A. Yagola, "Numerical Methods for the Solution of Ill-Posed Problems," Netherlands: Springer Netherlands. Retrieved 9 August 2018. ISBN 079233583X

[25] N.D. Assimakis, "Kalman Filter Gain Elimination in Linear Estimation.” Pages 183-188, Volume 2, 2020, ISSN: 2692-5079, www.engw.org

[26] N. Assimakis, M. Adam, and G. Tziallas, "Lainiotis Information Filter." Pages 270-273, Engineering World, Vol. 2, 2020, ISSN:2692-5079, www.engw.org

[27] O. Fomin, V. Pavlenko, "Construction of Diagnostic Features Space Using Volterra Kernels Moments”, The 20th International Conference on Methods and Models in Automation and Robotics (MMAR 2015), 24-27 August 2015, Międzyzdroje, Poland. , Publisher: IEEE, 2015. , pp. 1022-1027. - DOI: 10.1109/MMAR.2015.7284019. 
[28] A. Medvedev,O. Fomin, V. Pavlenko, and V. Speranskyy, "Diagnostic Features Space Construction using Volterra Kernels Wavelet Transforms," Proceedings of the 9th IEEE International Conference on Intelligent Data Acquisition and Advanced Computing Systems: Technology and Applications (IDAACS'2017), 21-23 September, 2017, Bucharest, Romania. Vol. 2, pp. 10771081.

[29] O. Fomin, A. Medvedev, and V. Pavlenko, "Technology of Intelligent Diagnostics based on Volterra Kernels Moments," Systems: Technology and Applications. Proc. of the $8^{\text {th }}$ IEEE Interrnational Conference IDAACS-2015, 24-26 September 2015, Warsaw, Poland, pp. 796-801.

[30] V. Vapnik, "The Nature of Statistical Learning Theory.” Springer-Verlag New York Inc., 2010.

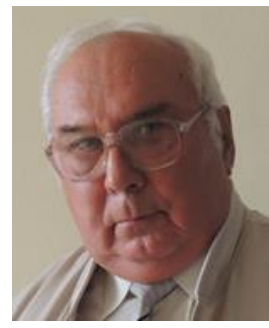

Vitaliy D. Pavlenko, DSc Eng., Professor (full), Senior Researcher, Institute Computer Systems in the Odessa National Polytechnic University (ONPU), Odessa, Ukraine.

Scopus Author ID: 54401442600

ORCID ID: 0000-0002-5655-4171

Academic Appointments: Associate Professor (1989-2013) and Professor (2013-present) Department of Computerized Control Systems, Institute Computer Systems, ONPU; Associate Professor (2001-2013) and Professor (2013-2017) Department of Maritime Radio Communication, National University "Odessa Maritime Academy".

Scientific activities: Member of the Specialized Academic Council for the Defense of Candidate's Dissertations in the specialty "Mathematical Modeling and Computational Methods" at ONPU, Odessa, Ukraine (2012-present) and of Doctoral Dissertations in the specialty "Information Technology" at the Black Sea National University named after Petro Mohyla, Mykolaiv, Ukraine (2013-present). Member of the Editorial Board of the International Scientific Journal "Applied Aspects of Information Technology" and "Informatics and Mathematical Methods in Simulation", ONPU, Odessa.

$\mathrm{He}$ is of the Supervision of $5 \mathrm{PhD}$ and $1 \mathrm{DSc}$ Eng dissertations. His research interests include modeling and simulation in IT and biomedical engineering applications, nonparametric identification of nonlinear dynamic systems and in theory of the Volterra series. Prof. Pavlenko have a Honorary Diploma of the Ministry of Education and Science of Ukraine (2005); Diploma of the Ministry of Education and Science of the Russian Federation (2005).

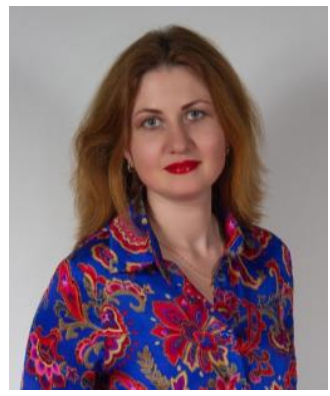

Tetiana V. Shamanina, MSC, Math Teacher and Deputy Director of Odessa Specialized School No 117, Odessa, Ukraine (2015-present).

ORCID ID: 0000-0002-3857-1867

The applicant of a scientific degree of candidate of Technical Sciences $(\mathrm{PhD})$ on Specialty "Mathematical modeling and computing methods" at the Department of Computerized Control Systems in the Odessa National Polytechnic University. Main Research Area "Mathematical modeling and computing methods".

Diploma of Mathematics, Speciality "Pedagogics and methodology of secondary education", qualification of master's degree of pedagogical education, teacher of mathematics, Nizhyn Gogol State University (2007).

Research activity: 2019-2021 experimental research and practical development is being carried out as part of the joint innovation project ONPU - Odessa Department of Junior Academy of Sciences of Ukraine - Odessa Specialized School No 117 "Eye Tracking in Psychology: Improving Cognitive Processes".

Took an active part in: the 5th International Scientific Conference the «Modeling of Nonlinear Processes and Systems», (MNPS-2020), Moscow State University of Technology "STANKIN", Moscow, Russia; the 9th International Scientific Conference "Modern problems of Mathematical Modeling, Forecasting and Optimization", (OPTIMA-2020), Kamenets-Podolsky National University named after Ivan Ogienko, Kamenets-Podolsky, Ukraine; 28th International Conference "Mathematics. Computer. Education" (MCE-2021), M.V. Lomonosov Moscow State University, Moscow, Russia.

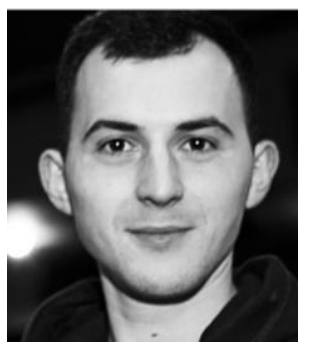

Vladyslav V. Chori, Postgraduate student Institute of Computer Systems in the Odessa National Polytechnic University, Odessa, Ukraine.

ORCID ID: 0000-0001-7823-8383

Graduated with a master's degree from Odessa National Polytechnic University with a degree in "Automation and computer-integrated technologies" with honors in 2018.

During his bachelor's and master's degrees he took part in the international student exchange program "Erasmus +" at the Lublin University of Technology - Faculty of Electrical Engineering and Computer Science (Lublin, Poland, 2016/2017 academic year) and at the University of Masaryk Faculty of Informatics (Brno, Czech Republic, 2017/2018 academic year), respectively.

Participant of the international competition "Formula Student" (Silverstone, UK, 2016) and the educational and scholarship program CIG R \& D Lab (Odessa, 2017/2018, 2018/2019) in the Startup Project "Eye Tracking Technology in Medical Research".

Participated in the following conferences: 5th International Scientific Conference the «Modeling of Nonlinear Processes and Systems», (MNPS-2020), Moscow State University of Technology "STANKIN", Moscow, Russia; 28th International Conference "Mathematics. Computer. Education" (MCE2021), M.V. Lomonosov Moscow State University, Moscow, Russia. 
Contribution of individual authors to the creation of a scientific article (ghostwriting policy)

Pavlenko Vitaliy carried out the simulation and the optimization.

Shamanina Tetiana has implemented the Algorithm (5) and calculations (10) in Python, organized and executed the experiments and experimental data processing of Section IV.

Chori Vladislav was responsible for the Statistics and building a Classifier.

This article is published under the terms of the Creative Commons Attribution License 4.0

https://creativecommons.org/licenses/by/4.0/deed.en_US 